\title{
Marginal-internal adaptation and fracture resistance of CAD/CAM crown restorations
}

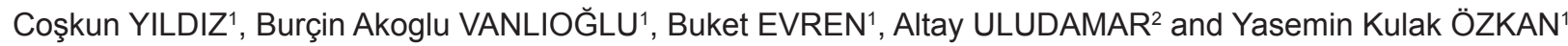 \\ ${ }^{1}$ University of Marmara, Department of Prosthetic Dentistry, Buyukciftlik Sok No:6, Nisantasi, Istanbul, Turkey \\ ${ }^{2}$ Private Practice, Ankara, Turkey \\ Corresponding author, Burçin Akoğlu VANLIOĞLU; E-mail: drburcinakoglu@hotmail.com
}

\begin{abstract}
This study evaluated the marginal adaptation and fracture resistance of CAD/CAM-fabricated zirconia crowns luted using different luting agents. Twenty crown restorations were produced using IPS ZirCAD zirconium oxide blocks (IZC), and another 20 using Lava zirconium oxide blocks (L). Ten teeth were used as control. Luting agents used were an etch-and-rinse adhesive (Variolink II) and a self-etch adhesive (Multilink Automix). Internal and marginal adaptation was evaluated using silicone replica technique. Fracture resistance was evaluated using a compression test. Marginal discrepancy was $89.26 \mu \mathrm{m}$ for L crowns and $88.84 \mu \mathrm{m}$ for IZC crowns, and difference was statistically insignificant. However, L crowns showed significantly larger axial and occlusal gaps than IZC crowns $(p<0.05)$. Fracture resistance of IZC-Multilink was higher than the other groups, although the difference was not significant. Results showed that CAD/CAM-fabricated crowns showed acceptable in vitro marginal discrepancies and fracture strengths.
\end{abstract}

Keywords: CAD/CAM, Crown, Internal adaptation, Marginal adaptation, Fracture resistance

\section{INTRODUCTION}

Metal-free, all-ceramic restorations have inundated the aesthetic dentistry field because of their high aesthetic appeal, excellent mechanical properties, and biocompatibility ${ }^{1-9)}$. Today, many framework structures for prosthetic restorations are fabricated using the computer aided design/computer-aided manufacturing (CAD/CAM) technology, where the construction of zirconia-based restorations uses a high-strength ceramic material for the framework to provide sufficient mechanical strength and resistance to chemical attack.

Another key factor that determines the clinical success of dental restorations is marginal adaptation ${ }^{7-9)}$. Poor marginal adaptation of restorations exposes the luting agent to the oral environment, rendering the restorations susceptible to microleakage and plaque retention. The larger the marginal discrepancy, the faster will be the rate of cement dissolution ${ }^{10)}$. Although the ideal marginal gap range of cemented restorations should be $25-40 \mu \mathrm{m}$, marginal gaps within this range are seldom achieved clinically. On the contrary, McLean and von Fraunhofer examined more than 1,000 crowns after five years of clinical service and stated that marginal discrepancies less than $120 \mu \mathrm{m}$ were clinically acceptable $^{11)}$.

It has been shown that milling of dense zirconia could yield restorations with high dimensional precision and high marginal fit $^{11-13)}$. If zirconia material is used in a semi-sintered porous state, it can be easily machined in a computer-aided manufacturing (CAM) unit. After machining, the framework has to be densely sintered to shrink to its original size ${ }^{14-16)}$. Volume changes occur during sintering, resulting in a linear sintering shrinkage of $15-30 \%$ and a concomitant increase in density ${ }^{17,18)}$. Other factors that cause marginal inaccuracies may arise from the CAD/CAM scanning, designing, and/or milling processes. Varied results on the marginal accuracy of different zirconia systems have been reported. For example, a mean marginal gap of $65 \mu \mathrm{m}$ was reported for fixed partial dentures (FPDs) milled from Lava zirconia (3M ESPE, Seefeld, Germany) in a semi-sintered state ${ }^{17)}$.

Fracture toughness of all-ceramic crowns is also an important property that has a direct correlation to their clinical success, and hence a keenly investigated subject in numerous in vitro studies ${ }^{19-21)}$. Regarding the effect of luting cements on the fracture resistance of all-ceramic crowns, it remains a controversial and widely debated issue. Based on several laboratory and clinical studies which investigated the fracture resistance and retentive strength of all-ceramic crowns, resin cements emerged as an optimal luting agent ${ }^{22,23)}$. Compared to conventional luting agents, resin cements exhibited enhanced mechanical, physical, and adhesive properties.

To simplify the luting procedure, a new group of selfetch and self-adhesive resin cements was introduced. These products contain highly acidic and hydrophilic monomers which demineralize and infiltrate enamel and dentin, resulting in strong bonding. Therefore, they do not require any conditioning or priming of tooth structure prior to bonding. For the cementation of zirconia-based crowns and fixed partial dentures, composite resin, glass ionomer, or resin-modified glass ionomer cements were recommended ${ }^{11}$.

The purpose of this study was to investigate the marginal and internal adaptation of CAD/CAM crowns fabricated using two commercial brands of partially stabilized zirconia systems, IPS ZirCAD (Ivoclar Vivadent AG, Schaan, Liechtenstein) and Lava Frame 
(3M ESPE, Seefeld, Germany). Crown restorations were luted using two different adhesive systems, etchand-rinse (Variolink II, Ivoclar Vivadent AG, Schaan, Liechtenstein) versus self-etch (Multilink Automix, Ivoclar Vivadent AG). Fracture resistance was evaluated by subjecting cemented CAD/CAM crowns to compressive loading. The two hypotheses of this study were: (1) Both zirconia systems would produce marginal gaps below 120 $\mu \mathrm{m}$; and (2) There would be no statistically significant differences in the marginal and internal adaptation and fracture resistance of both zirconia systems.

\section{MATERIALS AND METHODS}

\section{Selection of teeth}

Fifty intact, non-carious, unrestored human maxillary second premolars, extracted for periodontal reasons, were selected for this study. Immediately after extraction, teeth were scaled and immersed in a germfree $0.1 \%$ thymol solution at room temperature for 1 day. They were then stored in distilled water not longer than 3 months before use. Prior to testing, the size ( $<5 \%$ deviation) and quality (free of fillings, caries, and fractures) of teeth were examined under a light microscope at $\times 20$ magnification.

\section{Preparation of teeth for crown restoration}

Teeth were mounted individually in an acrylic resin (Meliodent, Heraeus Kulzer, Berkshire, UK) in a PVC ring. A dental surveyor (Kavo EWL, Typ 990, Kavo Elektrotechnisches Werk GmbH, Leutkirch im Allgau, Germany) was used to ensure that the long axis of tooth was perpendicular to the top surface of acrylic resin block. Each tooth was suspended in the middle of the ring with a $0.8-\mathrm{mm}$ orthodontic wire (Leowire round spring hard wire $0.8 \mathrm{~mm}$, Leowire s.p.a. Firenze, Italy) which engaged the tooth at cementoenamel junction $(\mathrm{CEJ})$ and which rested on the edges of the PVC ring.

Prior to preparing the teeth for crown restoration, two silicone impressions of each tooth were taken. One impression was used as an orientation aid for the wax-up of the crown, while the other was cut in a vestibulo-oral direction to assist in the removal of tooth structure.

All teeth were prepared according to a standardized protocol as follows: chamfer finish line was $1 \mathrm{~mm}$ above the CEJ; all sharp or internal line angles were rounded; preparation margins were not bevelled. Minimum layer thickness of each ceramic material as specified by the respective manufacturers was observed for all crown restorations. Bulk reduction was carried out using large-grit diamonds, while finishing was completed using finer diamonds. All teeth were prepared by one dental technician, and standardized crown preparation was accomplished by fixing the dental handpiece in a parallelometer.

\section{Fabrication of CAD/CAM zirconia crowns}

Putty-wash impressions (Virtual, Ivoclar Vivadent AG, Schaan, Liechtenstein) of crown preparations were made and used for laboratory manufacturing of crown restorations. Twenty crowns were produced using CAD/ CAM technology with IPS ZirCAD zirconium oxide blocks (IZC; Ivoclar Vivadent, Schaan, Liechtenstein), and another 20 crowns with Lava Frame zirconium oxide blocks (L; 3M ESPE, Seefeld, Germany). Both IZC and L crowns had a zirconia shoulder with rounded internal line angles (Fig. 1). CAD/CAM zirconia cores of $0.5 \mathrm{~mm}$ thickness were milled in the pre-sintered stage and then veneered with a hand-layered porcelain (IPS e.max, Ivoclar Vivadent, Schaan, Liechtenstein) at a commercial dental laboratory (Optimal Dental Laboratory, Istanbul, Turkey).

Both CAD/CAM scanner and milling machine were calibrated at the beginning of the study, and then recalibrated as prompted by the computer software. For milling, a new set of CAD/CAM milling burs was used for each group of pre-sintered IZC and L blocks.

\section{Cementation of CAD/CAM zirconia crowns}

Luting space was set at $25 \mu \mathrm{m}$. Fit of crowns was evaluated by the same dental technician, and internal adjustments were performed using diamond finishing burs. All frameworks were returned to their respective dies and manipulated to ensure complete seating. In the event of incomplete seating, additional framework adjustment was performed using a standard protocol according to published literature and clinical practice.

To identify areas that needed correction, a lipstick was applied to the die and the framework was placed on the latter without force. Red spots inside the framework were removed using a red ring diamond ball instrument (Komet 8801.016, Brasseler, GA, USA) with a watercooling spray. This procedure was repeated until the

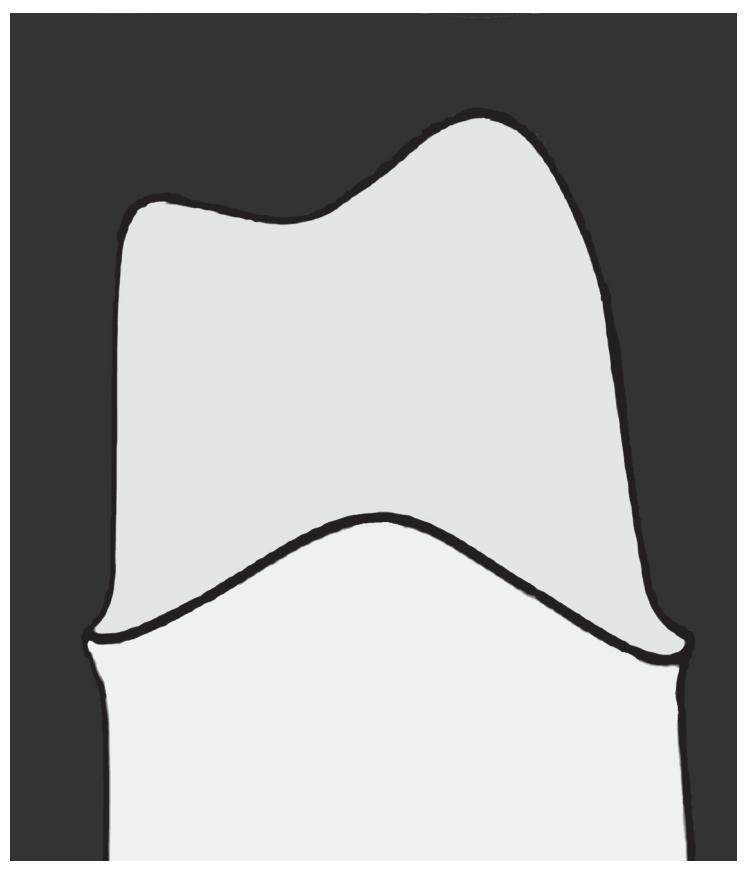

Fig. 1 Schematic presentation of framework design. 

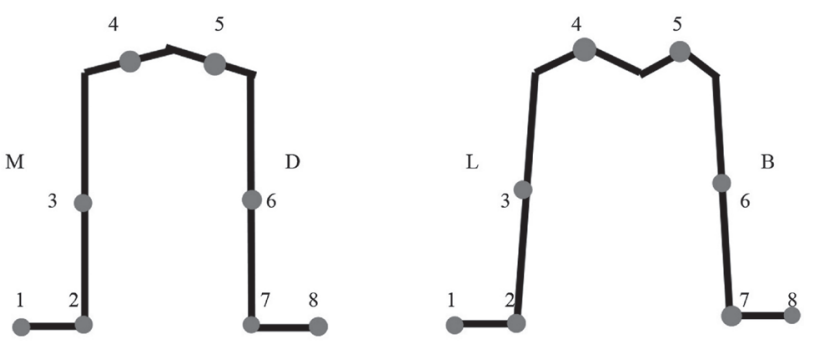

Fig. 2 Mesio-distal and bucco-lingual sections showing the locations of the measuring points.

red indicator spots disappeared, and that a uniform and even contact of the framework on the die was achieved. After each refinement, a steam cleaner was used to remove the lipstick dye from the die.

\section{Internal and marginal adaptation evaluation}

Internal and marginal adaptation of the crown to the prepared tooth was evaluated using silicone replica technique. Replicas of tooth specimens were sectioned buccolingually and mesiodistally, and fit on silicone replicas was examined using a light microscope (Leica Microscopes Ltd., Cambridge, UK) at $\times 200$ magnification. Eight measurement points were assessed for each of the five sections (Fig. 2). In each specimen, 40 measurements were evaluated, amounting to a total of 1,600 measurements for both zirconia systems (40 measurements per specimen $\times 20$ crowns per zirconia system $\times 2$ zirconia systems). By calculating the mean values of measurement points, the total mean values of occlusal, axial, internal-marginal, and marginal adaptations were obtained. Statistical analysis was performed using SPSS for Windows, version 11.0. (SPSS, Chicago, IL, USA). Results were compared using independent samples $t$-test at a significance level of 0.05 .

\section{Fracture resistance evaluation}

Before cementation, all the prepared tooth surfaces were cleaned with pumice and rinsed with water. Ten crowns of each zirconia system were cemented using an etchand-rinse adhesive and a high-viscosity composite resin cement (Syntac/Variolink II, Ivoclar Vivadent, Schaan, Liechtenstein); the remaining 10 crowns were cemented with a self-etch adhesive and a dual-cure universal resin cement (Multilink Automix, Ivoclar Vivadent, Schaan, Liechtenstein).

Cement was applied to the internal surface of each crown. Simulating the clinical procedure, the crown was seated on the prepared tooth with firm finger pressure. Excess cement was removed using a hand instrument. Each cemented crown was light-cured for $40 \mathrm{~s}$ from the occlusal, buccal, and lingual sides. After crown cementation, all specimens were stored in distilled water at room temperature for 2 days until the compression test.
To evaluate fracture resistance, each specimen was mounted in a custom-made device to ensure that loading force was applied on the occlusal surface and parallel to the long axis. Specimens were then subjected to compressive loading using a 3.5-mm-diameter steel ball in a universal testing machine (Testometric Micro 500, Testometric Co. Ltd., Lancashire, UK) at a crosshead speed of $0.5 \mathrm{~mm} / \mathrm{min}$ until failure occurred. For each specimen, maximum load to fracture was measured and indicated on a digital display. Results were analyzed by analysis of variance and Tukey's test using a software package (SPSS 13.0 for Windows, SPSS Inc., IL, USA).

Fractured crowns were recovered from the device, and fracture surfaces were visually inspected for failure pattern type.

\section{RESULTS}

\section{Marginal and internal adaptation}

Table 1 shows the means and standard deviations of marginal and internal gaps in each group. Marginal discrepancy was $89.26 \mu \mathrm{m}$ for L crowns and $88.84 \mu \mathrm{m}$ for IZC crowns. Both marginal and marginal-internal gaps between L and IZC crowns showed statistically insignificant differences. Significantly larger axial and occlusal gaps were found for L than for IZC $(p=0.001)$. Axial gaps were $106.21 \mu \mathrm{m}$ and $77.97 \mu \mathrm{m}$ for L and IZC respectively. Occlusal gaps were $240.3 \mu \mathrm{m}$ and 142.54 $\mu \mathrm{m}$ for L and IZC respectively.

\section{Fracture resistance}

Table 2 shows the means and standard deviations of failure loads obtained from the fracture test. Mean failure load of teeth restored with IZC cemented with Multilink $(2,202.19 \pm 276.97 \mathrm{~N})$ was higher than that of IZC cemented with Variolink $(2,074.74 \pm 347.30 \mathrm{~N})$. However, failure load of $\mathrm{L}$ cemented with Multilink $(2,166.03 \pm 357.62 \mathrm{~N})$ was not significantly different $(p>0.05)$ from that of $\mathrm{L}$ cemented with Variolink $(2,112.87 \pm 529.30 \mathrm{~N})$. Control unrestored teeth had a mean failure of load of $1,395.68 \pm 485.27 \mathrm{~N}$, which was significantly lower than the restored teeth $(p<0.05)$.

Comparison of the resin cements showed that crowns (both IZC and L; $n=20$ ) cemented with Multilink $(2,184.11 \pm 311.87 \mathrm{~N})$ had a higher mean failure load than cementation with Variolink $(2,093.80 \pm 436.15$ $\mathrm{N})$. However, the difference was not statistically significant.

\section{Failure patterns}

Table 3 shows the three categories of failure patterns as follows: Porcelain $(\mathrm{P})$, Porcelain+Core $(\mathrm{P}+\mathrm{C})$, and Porcelain+Core+Teeth $(\mathrm{P}+\mathrm{C}+\mathrm{T})$. Twenty-two specimens exhibited porcelain fracture, 10 exhibited porcelain+core fracture, and eight exhibited porcelain+core+teeth fracture.

For ' $\mathrm{P}$ ' failure pattern, the fractured surfaces of both L and IZC crowns were clean and smooth. No irregularities were detected upon visual inspection without magnification. The porcelain veneer delaminated 
Table 1 Mean and standard deviation values of marginal, internal-marginal, axial, and occlusal discrepancies for L and IZC $(\mu \mathrm{m})$

\begin{tabular}{lcccc}
\hline & Marginal Points 1-8 & Internal-marginal & Axial & Occlusal \\
\hline L & $89.26(36.06)$ & $86.82(31.47)$ & $106.21(26.86)$ & $240.3 \quad(79.8)$ \\
IZC & $88.84(28.91)$ & $87.31(22.11)$ & $77.97(18.16)$ & $142.54(39.95)$ \\
Total & $89.05(32.26)$ & $87.06(26.85)$ & $92.09(26.77)$ & $191.42(79.57)$ \\
Significance & 0.968 & 0.955 & 0.001 & 0.001 \\
\hline
\end{tabular}

Table 2 Mean and standard deviation values of load at fracture

\begin{tabular}{ll}
\hline \multicolumn{1}{c}{ Group } & Failure load (N) \\
\hline L-Multilink & $2,166.03(357.62)$ \\
L-Variolink & $2,112.87(529.31)$ \\
IZC-Multilink & $2,202.19(276.97)$ \\
IZC-Variolink & $2,074.74(347.3)$ \\
Control & $1,395.68(485.28)$ \\
Total & $1,990.30(496.58)$ \\
\hline
\end{tabular}

about 1-2 $\mathrm{mm}$ from the fracture margin of the core for both L and IZC crowns. The surface where the porcelain veneer detached from the core appeared smooth with no residual porcelain detected. Comparison of the resin cements showed that cementation with Multilink (both $\mathrm{L}$ and IZC) showed less 'P' fractures than cementation with Variolink.

\section{DISCUSSION}

Dental CAD/CAM systems have been available for nearly 20 years. Emergence of this technology engendered a rapid development of diverse equipment and materials available for the fabrication - and proliferation - of allceramic prosthese ${ }^{1,2,4)}$. Notably, the availability of CAD/ CAM processing permitted the use of polycrystalline zirconia coping and framework materials for all-ceramic fixed partial dentures in the posterior region. In the present study, IPS ZirCAD (Ivoclar Vivadent) and Lava Frame (3M ESPE) were used for crown fabrication and they were milled in partially-stabilized pre-sintered state.

\section{Marginal and internal adaptation}

According to published literature, marginal gaps of $\mathrm{CAD} / \mathrm{CAM}$ all-ceramic reportedly ranged between 53 and $108 \mu \mathrm{m}$, depending on cement film thickness ${ }^{24,25)}$. As for conventional all-ceramic crowns, marginal gaps reportedly ranged between 123 and $154 \mu \mathrm{m}^{25)}$. In the present study, marginal gaps were $89.26 \mu \mathrm{m}$ for $\mathrm{L}$ crowns and $88.84 \mu \mathrm{m}$ for IZC crowns. Both CAD/CAM zirconia systems demonstrated clinically acceptable marginal
Table 3 Failure pattern distribution of specimens, where $\mathrm{P}$ : Porcelain; $\mathrm{P}+\mathrm{C}$ : Porcelain+Core; $\mathrm{P}+\mathrm{C}+\mathrm{T}$ : Porcelain+Core+Teeth

\begin{tabular}{cccc}
\hline Group & $\mathrm{P}$ & $\mathrm{P}+\mathrm{C}$ & $\mathrm{P}+\mathrm{C}+\mathrm{T}$ \\
\hline L-Multilink & 4 & 4 & 2 \\
L-Variolink & 7 & 1 & 2 \\
IZC-Multilink & 4 & 4 & 2 \\
IZC-Variolink & 7 & 1 & 2 \\
\hline
\end{tabular}

discrepancies in vitro.

On axial and occlusal gaps, L exhibited significantly larger values $(p<0.05)$ than IZC; nonetheless, they were well within the clinically acceptable range. This large contrast in internal fit between L and IZC crowns could be due to differences in material properties, sintering shrinkage, and dimensional stability between the two zirconia systems. One in vitro study showed that a lack of precision in internal fit could heighten the risk of veneering fractures ${ }^{26)}$.

Tuntiprawon and Wilson ${ }^{27)}$ reported that all-ceramic crowns exhibited greater fracture strength when cement thickness at the axial wall was $73.0 \mu \mathrm{m}$. When cement thickness was increased to $122.0 \mu \mathrm{m}$, lower fracture strength was obtained without any significant improvement in seating. In this study, a thicker cement layer at the axial wall in $\mathrm{L}$ group might have adverse impact on the clinical performance and fracture resistance of the restorations.

At the occlusal surface, the cement thicknesses of both L and IZC crowns were markedly larger than the set value of $25 \mu \mathrm{m}$. These large discrepancies could be due to the shrinkage of zirconia blanks subjected to postmachining sintering.

For both L and IZC crowns, their fit was less accurate in the internal regions than at the marginal area. These results agreed with findings of previous studies regarding $\mathrm{CAD} / \mathrm{CAM}$ crowns ${ }^{14,17)}$. Crown fabrication by the $\mathrm{CAD} /$ CAM technique involves scanning, software design, and milling processes, where each step contributes to the overall fit of the crown. At the same time, the process of grinding down a tooth in preparation for a crown also 
affects the latter's internal adaptation. The narrowest possible diameter of tooth preparation is determined by the smallest diameter of the bur used for grinding the internal surface. Thus, in structures smaller than the narrowest bur diameter, more tooth substance may be removed than necessary. This may then result in larger internal gaps than mandatory for a good fit ${ }^{17)}$.

\section{Fracture resistance}

Highly lauded for their mechanical and optical properties, zirconia-based restorations may be an appealing alternative to metal-ceramic restorations as they have the potential to withstand physiological occlusal forces applied in the posterior region ${ }^{28}$. However, the failure rate of posterior all-ceramic crowns was reported to be $3-4 \%$ per year, prevalently due to cohesive failure in the porcelain veneer ${ }^{29}$. In a 5 -year clinical study on zirconia frameworks for posterior fixed partial dentures, no framework failures were observed but chipping of the porcelain veneer accounted for $15.2 \%$ of the failures ${ }^{18}$. In another clinical study on 204 zirconia single crowns among 161 patients, $78 \%$ of the crowns were placed on premolars and molars ${ }^{30}$. At the 3rd year's follow-up, there were no zirconia core fractures but two incidences of feldspar porcelain veneer fracture.

In the present in vitro study, 22 specimens exhibited porcelain fracture and the surface where the porcelain veneer delaminated from the core appeared smooth with no residual porcelain detected. A wide spectrum of factors reportedly contributed to this chipping or cracking $28,29,31,32)$ : framework thickness, inappropriate framework support for the veneer, mechanical insufficiency of veneering porcelain, thermal misfit between veneering porcelain and zirconia framework and hence development of residual thermal stresses in porcelain veneer, presence of unfavorable shear forces between framework and porcelain veneer or occlusal stresses. For example, Reich et $a l .{ }^{33)}$ reported that when framework thickness of single crowns was reduced from 0.5 to $0.3 \mathrm{~mm}$, fracture resistance was unfavorably reduced by $35 \%$. To avoid porcelain fractures, suggestions to improve zirconia coping design included uniform porcelain veneer layer thickness and improved support for occlusal and lateral loading as well as compressive loading ${ }^{31)}$.

For zirconia-based crowns, most fractures occurred in the veneering porcelain. Nonetheless, zirconia cores can be veneered with a heat-pressed or sintered veneering material as they did not significantly affect the fatigue and fracture properties of zirconia cores ${ }^{21}$. In the present study, IPS e.max Ceram material was used to veneer the zirconia-based crowns using a handlayering technique.

\section{Effect of luting agents on fracture resistance}

A strong, durable resin bond provides high retention, improves marginal adaptation, prevents microleakage, and increases fracture resistance of the restored tooth and the restoration. The composition and physical properties of zirconium oxide-based ceramics differ substantially from silica-based ceramics, such that conventional acid-etching has no positive effects on the resin bond to zirconium oxide ceramics ${ }^{34)}$. As resin bonding to high-strength ceramics is less predictable, alternative bonding techniques are required to achieve a strong, long-term, durable resin bond. Primers containing phosphoric acid monomer, such as Metal/ Zirconia Primer (Ivoclar Vivadent), could establish a chemical bond to the surfaces of oxide ceramics. Hence, they are deemed as an alternative to promote adhesion to oxide ceramics such as zirconia.

In the present study, two different adhesive systems (self-etch versus etch-and-rinse) were investigated for their effects on the fracture resistance of two different CAD/CAM partially stabilized zirconia systems. Cementation of zirconia crowns with self-etch adhesive (Multilink) resulted in higher fracture resistance than cementation with etch-and-rinse adhesive (Variolink), but there were no significant differences. Cementation with Multilink also resulted in less porcelain veneer fractures than cementation with Variolink.

\section{Limitations of an in vitro study}

In the present in vitro study, standardized and optimized conditions were provided for every experimental procedure, from tooth preparation and crown cementation to internal fit and fracture resistance evaluations. Therefore, the results of the present study depicted the production and precision of CAD/CAM zirconia crowns under ideal conditions in a controlled and predictable environment, but not a faithful reflection of those conditions encountered in daily clinical care.

Limitations of the present study included:

1. Gap dimensions were measured using the silicone replica technique. This meant that precision of fit was measured at only 35 defined points, which might not fully represent the complete fit.

2. Fit of the CAD/CAM zirconia crowns was recorded before cementation to compare only the fabrication procedures. Besides, cross-sectioning might have caused some damage to the specimens after cementation.

\section{CONCLUSIONS}

Based on the results of this study, the following conclusions were drawn:

1. Differences in precision of fit between the two CAD/CAM zirconia systems tested in this study depended on the region being evaluated.

2. Both L and IZC systems demonstrated acceptable in vitro marginal discrepancies, with no statistically significant differences $(p>0.05)$.

3 . The IZC system showed significantly lower axial and occlusal discrepancies.

4. Cementation with self-etch Multilink for both L and IZC systems resulted in higher fracture resistance than cementation with Variolink, but the differences were not statistically significant $(p>0.05)$.

5. Unrestored control teeth exhibited significantly 
lower fracture resistance than the restored teeth.

6. As per the most common technical complication encountered for CAD/CAM zirconia frameworks, porcelain veneer fracture was the predominant failure pattern in this study.

\section{REFERENCES}

1) Barnfather KD, Brunton PA. Restoration of the upper dental arch using Lava all-ceramic crown and bridgework. Br Dent J 2007; 202: 731-735.

2) Coli P, Karlsson S. Precision of a CAD/CAM technique for the production of zirconium dioxide copings. Int $J$ Prosthodont 2004; 17: 577-580.

3) Della Bona A, Mecholsky JJ Jr, Anusavice KJ. Fracture behavior of lithia disilicate- and leucite-based ceramics. Dent Mater 2004; 20: 956-962.

4) Devaud V. Guidelines for success with zirconia ceramics: the changing standards. Pract Proced Aesthet Dent 2005; 17: 508-510.

5) Raigrodski AJ. All-ceramic full-coverage restorations: concepts and guidelines for material selection. Pract Proced Aesthet Dent 2005; 17: 249-256.

6) Sorensen JA, Choi C, Fanuscu MI, Mito WT. IPS empress crown system: three-year clinical trial results. J Calif Dent Assoc 1998, 26: 130-136.

7) Sorensen JA, Kang SK, Torres TJ, Knode H. In-ceram fixed partial dentures: three-year clinical trial results. J Calif Dent Assoc 1998; 26: 207-214.

8) Luthardt RG, Holzhuter MS, Rudolph H, Herold V, Walter MH. CAD/CAM-machining effects on Y-TZP zirconia. Dent Mater 2004; 20: 655-662.

9) Tinschert J, Natt G, Hassenpflug S, Spiekermann H. Status of current $\mathrm{CAD} / \mathrm{CAM}$ technology in dental medicine. Int $\mathrm{J}$ Comput Dent 2004; 7: 25-45.

10) Jacobs MS, Windeler AS. An investigation of dental luting cement solubility as a function of the marginal gap. $J$ Prosthet Dent 1991; 65: 436-442.

11) McLean JW, von Fraunhofer JA. The estimation of cement film thickness by an in vivo technique. Br Dent J 1971; 131: 107-111.

12) Schaerer P, Sato T, Wohlwend A. A comparison of the marginal fit of three cast ceramic crown systems. J Prosthet Dent 1988; 59: 534-542.

13) Tinschert J, Natt G, Mautsch W, Spiekermann H, Anusavice KJ. Marginal fit of alumina- and zirconia-based fixed partial dentures produced by a CAD/CAM system. Oper Dent 2001; 26: 367-374.

14) Curtis AR, Wright AJ, Fleming GJ. The influence of simulated masticatory loading regimes on the bi-axial flexure strength and reliability of a Y-TZP dental ceramic. J Dent 2006; 34: 317-325.

15) Curtis AR, Wright AJ, Fleming GJ. The influence of surface modification techniques on the performance of a Y-TZP dental ceramic. J Dent 2006; 34: 195-206.

16) Kosmac T, Oblak C, Jevnikar P, Funduk N, Marion L. The effect of surface grinding and sandblasting on flexural strength and reliability of Y-TZP zirconia ceramic. Dent
Mater 1999; 15: 426-433.

17) Reich $S$, Wichmann M, Nkenke E, Proeschel P. Clinical fit of all-ceramic three-unit fixed partial dentures, generated with three different CAD/CAM systems. Eur J Oral Sci 2005; 113: 174-179.

18) Sailer I, Feher A, Filser F, Gauckler LJ, Luthy H, Hammerle $\mathrm{CH}$. Five-year clinical results of zirconia frameworks for posterior fixed partial dentures. Int J Prosthodont 2007; 20: 383-388.

19) Pallis K, Griggs JA, Woody RD, Guillen GE, Miller AW. Fracture resistance of three all-ceramic restorative systems for posterior applications. J Prosthet Dent 2004; 91:561-569.

20) Potiket N, Chiche G, Finger IM. In vitro fracture strength of teeth restored with different all-ceramic crown systems. J Prosthet Dent 2004; 92: 491-495.

21) Mörmann W, Wolf D, Ender A, Bindl A, Göhring T, Attin T. Effect of two self-adhesive cements on marginal adaptation and strength of esthetic ceramic CAD/CAM molar crowns. J Prosthodont 2009; 18: 403-410.

22) Hill EE. Dental cements for definitive luting: A review and practical clinical considerations. Dent Clin North Am 2007; 51: 643-658.

23) Ernst CP, Cohnen U, Stender E, Willershausen B. In vitro retentive strength of zirconium oxide ceramic crowns using different luting agents. J Prosthet Dent 2005; 93: 551-558.

24) Witkowski S, Komine F, Gerds T. Marginal accuracy of titanium copings fabricated by casting and CAD/CAM techniques. J Prosthet Dent 2006, 96: 47-52.

25) Grey NJ, Piddock V, Wilson MA. In vitro comparison of conventional crowns and a new allceramic system. J Dent 1993; 21: 47-51.

26) Rekow ED, Harsono M, Janal M, Thompson VP, Zhang G. Factorial analysis of variables influencing stress in allceramic crowns. Dent Mater 2006; 22: 125-132.

27) Tuntiprawon M, Wilson PR. The effect of cement thickness on the fracture strength of all-ceramic crowns. Aust Dent J 1995; 40: 17-21.

28) Komine F, Blatz MB, Matsumura H. Current status of zirconia-based fixed restorations. J Oral Sci 2010; 52: 531539.

29) Coelho PG, Silva NR, Bonfante EA, Guess PC, Rekow ED, Thompson VP. Fatigue testing of two porcelain-zirconia allceramic crown systems. Dent Mater 2009; 25: 1122-1127.

30) Örtorp A, Kihl ML, Carlsson GE. A 3-year retrospective and clinical follow-up study of zirconia single crowns performed in a private practice. J Dent 2009; 37: 731-736.

31) Kokubo Y, Tsumita M, Kano T, Fukushima S. The influence of zirconia coping designs on the fracture load of all-ceramic molar crowns. Dent Mater J 2011; 30: 281-285.

32) Fischer J, Stawarczyk B, Tomic M, Strub JR, Hammerle CH. Effect of thermal misfit between different veneering ceramics and zirconia frameworks on in vitro fracture load of single crowns. Dent Mater J 2007; 26: 766-772.

33) Reich S, Petschelt A, Lohbauer U. The effect of finish line preparation and layer thickness on the failure load and fractography of $\mathrm{ZrO}_{2}$ copings. J Prosthet Dent 2008; 99: 369376.

34) Blatz MB, Sadan A, Kern M. Resin-ceramic bonding: a review of the literature. J Prosthet Dent 2003; 89: 268-274. 